\title{
ON THE CRITICAL EXPONENT FOR $k$-PRIMITIVE SETS
}

\author{
TSZ HO CHAN, JARED DUKER LICHTMAN, AND CARL POMERANCE
}

\begin{abstract}
A set of positive integers is primitive (or 1-primitive) if no member divides another. Erdős proved in 1935 that the weighted sum $\sum 1 /(n \log n)$ for $n$ ranging over a primitive set $A$ is universally bounded over all choices for $A$. In 1988 he asked if this universal bound is attained by the set of prime numbers. One source of difficulty in this conjecture is that $\sum n^{-\lambda}$ over a primitive set is maximized by the primes if and only if $\lambda$ is at least the critical exponent $\tau_{1} \approx 1.14$.

A set is $k$-primitive if no member divides any product of up to $k$ other distinct members. One may similarly consider the critical exponent $\tau_{k}$ for which the primes are maximal among $k$-primitive sets. In recent work the authors showed that $\tau_{2}<0.8$, which directly implies the Erdős conjecture for 2-primitive sets. In this article we study the limiting behavior of the critical exponent, proving that $\tau_{k}$ tends to zero as $k \rightarrow \infty$.
\end{abstract}

\section{INTRODUCTION}

A set $A \subset \mathbb{Z}_{>1}$ is primitive if no member of $A$ divides another. Erdős [5] showed that for any primitive set $A$,

$$
\sum_{n \in A} \frac{1}{n \log n}<\infty
$$

In fact, his proof bounded the sum uniformly over all primitive sets A. Further, in 1988 he asked if the maximizer is the set of primes $A=\mathbb{P}$. This is now referred to as the Erdős conjecture for primitive sets:

$$
\text { For primitive A, we have } \sum_{n \in A} \frac{1}{n \log n} \leq \sum_{p \in \mathbb{P}} \frac{1}{p \log p}=1.6366 \cdots \text {, }
$$

The current record bound is $\sum_{n \in A} 1 /(n \log n)<e^{\gamma}=1.781 \cdots$ due to the second and third authors [10]. Here $\gamma$ is the Euler-Mascheroni constant.

A potential approach towards the Erdős conjecture is via integration. Namely, we have

$$
\sum_{n \in A} \frac{1}{n \log n}=\int_{1}^{\infty}\left(\sum_{n \in A} \frac{1}{n^{\lambda}}\right) d \lambda,
$$

and one might hope the integrand above is dominated by $\sum_{p} p^{-\lambda}$ for all $\lambda>1$. Note by a simple argument (see Lemma 1), if this inequality holds for an exponent $\lambda$, then it will continue to hold for all larger exponents $\lambda^{\prime}>\lambda$.

Date: December 3, 2020.

2010 Mathematics Subject Classification. Primary 11B75; Secondary 11A05, 05 C70.

Key words and phrases. primitive set, primitive sequence. 
However, the primes are not maximal among primitive sets with respect to logarithmic density (i.e., $\lambda=1$ ). Indeed, by Erdős [7] and Erdős, Sárközy, and Szemerédi [8],

$$
\sup _{\text {primitive }} \sum_{\substack{n \in A \\ n \leq x}} \frac{1}{n}=\left(\frac{1}{\sqrt{2 \pi}}+o(1)\right) \frac{\log x}{\sqrt{\log \log x}}
$$

where the maximizer is the set of positive integers with $\lfloor\log \log x\rfloor$ prime factors (with multiplicity). By contrast, the primes satisfy

$$
\sum_{p \leq x} \frac{1}{p}=\log \log x+O(1) .
$$

Later, Banks and Martin [1] obtained the full characterization that

$$
\sum_{\substack{n \in A \\ n \leq x}} n^{-\lambda} \leq \sum_{p \leq x} p^{-\lambda}
$$

for all primitive $A, x>1$, if and only if $\lambda \geq \tau_{1}:=1.1403 \ldots$, where $\tau=\tau_{1}$ is the unique real solution to the equation

$$
\sum_{p \in \mathbb{P}} p^{-\tau}=1+\left(1-\sum_{p \in \mathbb{P}} p^{-2 \tau}\right)^{1 / 2} .
$$

As such we call $\tau_{1}$ the critical exponent for primitive sets.

One may define a hierarchy of primitivity as follows. A 1-primitive set is primitive, and inductively for $k>1$, a $(k-1)$-primitive set is $k$-primitive if no member divides the product of $k$ distinct other members. That is, a set $A \subset \mathbb{Z}_{>1}$ is $k$-primitive if no member of $A$ divides any product of $j$ distinct other members, for any $1 \leq j \leq k 1$ Note that if (1.2) holds for all $\lambda>\tau$, then it holds for $\lambda=\tau$. Thus, one may similarly consider the critical exponent $\tau_{k}$ for which (1.2) holds for all $k$-primitive sets if and only if $\lambda \geq \tau_{k}$. Note that $\tau_{j} \geq \tau_{k}$ for $1 \leq j \leq k$.

Recently, the authors [4] proved $\tau_{2} \leq 0.7983$. In particular $\tau_{2}<1$, thereby establishing the Erdös conjecture in the case of 2-primitive sets.

Theorem 1 ([4]). For $\lambda \geq 0.7983$, we have

$$
\sum_{\substack{n \in A \\ n \leq x}} n^{-\lambda} \leq \sum_{p \leq x} p^{-\lambda}
$$

for all 2-primitive sets $A$ and $x \geq 2$. In particular, any 2-primitive set $A$ satisfies

$$
\sum_{n \in A} \frac{1}{n \log n} \leq \sum_{p} \frac{1}{p \log p} .
$$

In 1938, Erdős [6] first studied the maximal cardinality of 2-primitive sets (i.e., $\lambda=0$ ). The first author together with Győri and Sárközy [3] extended it to all $k \geq 2$, also see [2]

\footnotetext{
${ }^{1}$ In $[3, A$ is called $k$-primitive if no member of $A$ divides any product of $k$ distinct other members. These definitions only differ when $|A| \leq k$, and do not affect the critical exponent $\tau_{k}$, by Lemma 4 below.
} 
and [11]. Namely, there is an absolute constant $c>0$ such that

$$
\frac{1}{8 k^{2}} \frac{x^{\frac{2}{k+1}}}{(\log x)^{2}} \leq \sup _{k \text {-primitive } A} \sum_{\substack{n \in A \\ n \leq x}} 1-\sum_{p \leq x} 1 \leq c k^{2} \frac{x^{\frac{2}{k+1}}}{(\log x)^{2}}
$$

for $x$ sufficiently large. Here the lower bound is attained by some set $A^{\prime \prime}$ consisting of the primes in $\left(x^{1 /(k+1)}, x\right]$ and a size $x^{2 /(k+1)} / 8(k \log x)^{2}$ subset of products of $k+1$ primes in $\left(1, x^{1 /(k+1)}\right]$. In particular, the lower bound in (1.4) implies

$$
\sum_{\substack{n \in A^{\prime \prime} \\ n \leq x}} n^{-\lambda} \geq \sum_{x^{1 /(k+1)}<p \leq x} p^{-\lambda}+\frac{1}{x^{\lambda}} \frac{x^{2 /(k+1)}}{8(k \log x)^{2}}>\sum_{x^{1 /(k+1)}<p \leq x} p^{-\lambda}+\sum_{p \leq x^{1 /(k+1)}} p^{-\lambda},
$$

when $\lambda<1 / k$ and $x$ is sufficiently large. Hence we quickly deduce $\tau_{k} \geq 1 / k$.

Thus combining with Theorem 1, the critical exponent for 2-primitive sets lies in the interval

$$
\tau_{2} \in[0.5,0.7983] .
$$

It is an open question to determine the exact value of $\tau_{2}$, and perhaps characterize $\tau_{2}$ as a solution to some functional equation, as with (1.2) for $\tau_{1}$.

In light of this, it is natural to ask about the behavior of the decreasing sequence $\tau_{1} \geq$ $\tau_{2} \geq \tau_{3} \geq \cdots$, in particular whether $\tau_{k}$ tends to zero as $k \rightarrow \infty$. The main result of this article is to answer in the affirmative, with the following quantitative result.

Theorem 2. Let $p_{k}$ denote the $k$ th prime number. For any $k \geq 1$ and $\lambda \geq 1.5 / \log p_{k}$, we have

$$
\sum_{\substack{n \leq x \\ n \in A}} n^{-\lambda} \leq \sum_{p \leq x} p^{-\lambda}
$$

for all $k$-primitive sets $A$ and $x \geq 2$.

Thus, for $k \geq 1$,

$$
\frac{1}{k} \leq \tau_{k} \leq \frac{1.5}{\log p_{k}}
$$

Clearly the upper and lower bounds differ substantially, and it remains an unsolved problem to narrow this gap.

1.1. Generalizations. Upon closer inspection of the proofs in [2], [3], we observe the lower bound for (1.4) holds under a stronger notion of $k$-primitivity, namely, one forbids a member from dividing the product of $k$ other members, not necessarily distinct. Similarly, the upper bound in (1.4) holds even if one relaxes to only forbid a member from dividing the least common multiple (lcm) of $k$ other members.

Hence this naturally suggests the following generalizations. We say a set $A \subset \mathbb{Z}_{>1}$ is "strongly $k$-primitive" if no member divides the product of $k$ other members which are not necessarily distinct. Any strongly $k$-primitive set is $k$-primitive, but not vice versa. For example, $A=\{4,5,6\}$ is 2-primitive but not strongly 2-primitive. In the other direction, we say a set $A \subset \mathbb{Z}_{>1}$ is "lcm $k$-primitive" if no member divides the lcm of $k$ other members. 
Here, every $k$-primitive set is lcm $k$-primitive, but not vice versa. An example is $A=$ $\{4,6,10\}$ which is lcm 2-primitive, but not 2-primitive.

One can ask for critical exponents in the strong case and in the lcm case. Denote the former by $\tau_{k}^{(\mathrm{s})}$ and the latter by $\tau_{k}^{(\mathrm{lcm})}$. By the above comments, for each $k \geq 2$ we have

$$
\frac{1}{k} \leq \tau_{k}^{(\mathrm{s})} \leq \tau_{k} \leq \tau_{k}^{(\mathrm{lcm})}
$$

From these definitions, two natural questions arise: Is there a better upper bound for $\tau_{k}^{(\mathrm{s})}$ than that afforded by Theorem 2]. Is there an upper bound for $\tau_{k}^{(\mathrm{lcm})}$ that is $o(1)$ as $k \rightarrow \infty$ ?

We make progress on these two questions by proving the following two theorems.

Theorem 3. For any $k \geq 1 \tau_{k}^{(\mathrm{lcm})} \leq 1.7 / \log p_{k}$. In addition, $\tau_{2}^{(\mathrm{lcm})} \leq 1$, so the Erdös conjecture is true for lcm 2-primitive sets.

For the $\tau_{k}^{(\mathrm{s})}$ case we prove a considerably stronger inequality.

Theorem 4. For $k \geq 2$ we have $\tau_{k}^{(\mathrm{s})} \leq(3 \log k) / k$.

Thus,

$$
\frac{1}{k} \leq \tau_{k}^{(\mathrm{s})} \leq \frac{3 \log k}{k}
$$

for all $k \geq 2$. It would be nice to so sharpen the inequalities for $\tau_{k}$ and $\tau_{k}^{(\mathrm{lcm})}$.

\section{Preliminary Lemmas}

Lemma 1. Take sets $A, B \subset \mathbb{R}_{>1}$. Suppose $\lambda \geq 0$ satisfies $I_{\lambda}(x) \geq 0$ for all $x>1$, where

$$
I_{\lambda}(x):=\sum_{\substack{a \in A \\ a \leq x}} a^{-\lambda}-\sum_{\substack{b \in B \\ b \leq x}} b^{-\lambda} .
$$

Then $I_{\lambda^{\prime}}(x) \geq 0$ for all $\lambda^{\prime} \geq \lambda, x>1$.

Proof. By partial summation,

$$
I_{\lambda^{\prime}}(x)=x^{\lambda-\lambda^{\prime}} I_{\lambda}(x)+\left(\lambda^{\prime}-\lambda\right) \int_{1}^{x} u^{\lambda-\lambda^{\prime}-1} I_{\lambda}(u) d u .
$$

Hence if $I_{\lambda_{k}}(x) \geq 0$ for all $x>1$, it then follows $I_{\lambda^{\prime}}(x) \geq 0$ for all $\lambda^{\prime} \geq \lambda$ as claimed.

Lemma 2. Let

$$
\lambda_{1}=1.2, \quad \lambda_{2}=0.8, \quad \text { and } \quad \lambda_{k}=2.625 \prod_{i=1}^{k}\left(1-\frac{1}{p_{i}}\right) \text { for } k \geq 3 .
$$

Then

$$
\lambda_{k}>\frac{1.45}{\log p_{k}} \text { for } k \geq 62, \quad \lambda_{k}<\frac{1.5}{\log p_{k}} \text { for } k \geq 1
$$

In addition, let

$$
\mu_{1}=8 / 7 \quad \text { and } \quad \mu_{k}=3 \prod_{i=1}^{k}\left(1-\frac{1}{p_{i}}\right) \text { for } k \geq 2 \text {. }
$$


Then

$$
\mu_{k}>\frac{1.65}{\log p_{k}} \text { for } k \geq 47, \quad \mu_{k}<\frac{1.7}{\log p_{k}} \text { for } k \geq 1 .
$$

Proof. One can verify the lemma for $p_{k} \leq 2,000$ by direct computation. For larger $p_{k}$ we use (3.25) of Rosser and Schoenfeld [12] with the Euler-Mascheroni constant $\gamma=0.57721 \ldots$, getting

$$
\lambda_{k} \geq \frac{2.625 e^{-\gamma}}{\log p_{k}}\left(1-\frac{1}{2 \log ^{2} p_{k}}\right) \geq \frac{2.625 e^{-0.57722}}{\log p_{k}}\left(1-\frac{1}{2 \log ^{2} 2,000}\right) \geq \frac{1.45}{\log p_{k}},
$$

which gives the lower bound for $\lambda_{k}$. The lower bound for $\mu_{k}$ follows in the same way. For the upper bound, by (3.26) of Rosser and Schoenfeld [12] we have

$$
\lambda_{k}<\frac{2.625 e^{-.57721}}{\log p_{k}}\left(1+\frac{1}{2 \log ^{2} 2,000}\right)<\frac{1.5}{\log p_{k}} .
$$

Again, the upper bound for $\mu_{k}$ follows in the same way. This completes the proof.

Lemma 3. For $0<\lambda<1$ and $x \geq 41$,

$$
x^{1-\lambda}\left(1-\frac{1}{\log x}\right) \leq \sum_{p \leq x} \frac{\log p}{p^{\lambda}} \leq \frac{1.01624}{1-\lambda} x^{1-\lambda} .
$$

Proof. By partial summation,

$$
\sum_{p \leq x} \frac{\log p}{p^{\lambda}}=\int_{2^{-}}^{x} \frac{d \theta(u)}{u^{\lambda}}=\frac{\theta(x)}{x^{\lambda}}+\lambda \int_{2}^{x} \frac{\theta(u)}{u^{\lambda+1}} d u
$$

where $\theta(x)=\sum_{p \leq x} \log p$. The lemma follows from (3.16) and (3.32) in Rosser and Schoenfeld

$$
x\left(1-\frac{1}{\log x}\right)<\theta(x) \text { for } x \geq 41
$$

and

$$
\theta(x)<1.01624 x \text { for } x>0 \text {. }
$$

For a set $A$ of integers, let $\mathcal{P}(A)$ denote the set of primes that divide some member of $A$.

Lemma 4. Let $A$ be an lcm $k$-primitive set with $k \geq 2$. If $|\mathcal{P}(A)| \leq k$, then $|A| \leq|\mathcal{P}(A)|$ and for all $\lambda \geq 0$,

$$
\sum_{n \in A} n^{-\lambda} \leq \sum_{p \in \mathcal{P}(A)} p^{-\lambda} .
$$

Also, if $k<|\mathcal{P}(A)|<2 k$, then $|A| \leq|\mathcal{P}(A)|+1$.

Proof. Let $v_{p}(n)$ denote the exponent on $p$ in the prime factorization of $n$, so that $p^{v_{p}(n)} \| n$. For each $p \in \mathcal{P}(A)$ let $n_{p}$ be the element $n \in A$ with $v_{p}(n)$ maximal (breaking ties arbitrarily), and let $A^{*}=\left\{n_{p}: p \in \mathcal{P}(A)\right\}$. Thus $\left|A^{*}\right| \leq|\mathcal{P}(A)|$.

Suppose $|\mathcal{P}(A)| \leq k$. Then any $n \in A \backslash A^{*}$ would satisfy $n \mid \operatorname{lcm}\left(A^{*}\right)$, contradicting $A$ as lcm $k$-primitive. Thus, $A^{*}=A$ and $|A| \leq|\mathcal{P}(A)| \leq k$. Next, $|A| \leq k$ implies each $n \in A$ 
has $n \nmid \operatorname{lcm}(A \backslash\{n\})$. Thus, each $n \in A$ has a prime factor $p$ with $v_{p}(n)>v_{p}(m)$ for all $m \in A \backslash\{n\}$, so the map, call it $f$, where $f(n)=p \mid n$ is injective on $A$. Hence we conclude

$$
\sum_{n \in A} n^{-\lambda} \leq \sum_{n \in A} f(n)^{-\lambda} \leq \sum_{p \in \mathcal{P}(A)} p^{-\lambda}
$$

Also, suppose $N=|\mathcal{P}(A)|, k<N<2 k$, and there exist distinct $n, n^{\prime} \in A \backslash A^{*}$. Without loss, the subset $P=\left\{p \in \mathcal{P}(A): v_{p}(n) \geq v_{p}\left(n^{\prime}\right)\right\}$ contains at least half of the primes in $\mathcal{P}(A)$, i.e., $|P| \geq\left\lceil\frac{N}{2}\right\rceil$. Hence

$$
n^{\prime} \mid \operatorname{lcm}\left(\left\{n_{p}: p \notin P\right\} \cup\{n\}\right),
$$

which is an lcm of $1+N-\lceil N / 2\rceil$ elements. It is easy to see this number is $\leq k$, thus contradicting $A$ as lcm $k$-primitive. This implies $|A| \leq N+1$.

\section{ThEOREM FOR $k$-PRIMITIVE SETS}

In this section we prove Theorem 2, Recall the numbers $\lambda_{k}$ in Lemma 2, By that lemma it suffices to prove the following theorem.

Theorem 5. Let $A$ be a $k$-primitive set. For each $k \geq 1$ we have

$$
\sum_{\substack{a \in A \\ a \leq x}} a^{-\lambda_{k}} \leq \sum_{\substack{p \in \mathcal{P}(A) \\ p \leq x}} p^{-\lambda_{k}}
$$

for any $x>1$.

Since $\lambda_{1}=1.2, \lambda_{2}=0.8$, the theorem holds for $k=1,2$, so we may assume that $k \geq 3$ and that the theorem holds for $(k-1)$-primitive sets.

We partition $A$ into primes $S$ and composites $T$. Note by primitivity, the primes in $S$ and $\mathcal{P}(T)$ are disjoint. We thus may cancel the contribution of $p \in S$ from both sides of (3.1) and so reduce Theorem 5 to the case $A=T$ where every member is composite.

We may assume that

$$
\sum_{t \in T_{p}} t^{-\lambda}>p^{-\lambda} \text { for all } p \in \mathcal{P}(T)
$$

since if this fails for some $p$, the theorem for $T \backslash T_{p}$ implies the theorem for $T$. An immediate consequence is that

$$
\left|T_{p}\right| \geq 2 \text { for all } \quad p \in \mathcal{P}(T) .
$$

Further, it suffices to assume that $\mathcal{P}(T)$ consists of an initial list of primes, say

$$
\mathcal{P}(T)=\mathbb{P} \cap(1, Y] \text { for some } Y \geq 2 .
$$

Indeed, if not, suppose $q$ is the smallest prime outside $\mathcal{P}(T)$, and let $p \in \mathcal{P}(T)$ be the smallest prime with $p>q$. Then by (3.2),

$$
0<(p / q)^{\lambda}\left(\sum_{t \in T_{p}} t^{-\lambda}-p^{-\lambda}\right) \leq \sum_{t^{\prime} \in T_{q}^{\prime}}\left(t^{\prime}\right)^{-\lambda}-q^{-\lambda},
$$

where $T^{\prime}$ is the ( $k$-primitive) image of $T$ under the automorphism of $\mathbb{N}$ induced by swapping $q \leftrightarrow p$. Hence the proof for $T$ will follow from that of $T^{\prime}$. 
For an integer $t>1$ let $Q(t)$ denote the largest prime power factor of $t$, which is possibly a prime to the first power. We first handle those $t \in T$ with $Q(t)<t^{\theta}$ for an appropriate choice of $\theta$.

Lemma 5. Let $k \geq 2$ and let $0<\theta \leq 1 / k$. Suppose $T$ is $\operatorname{lcm~} k$-primitive with $Q(t)<t^{\theta}$ for each $t \in T$. Let $z \geq 2$, and let $N(z)$ be the number of members of $T$ up to $z$. Then

$$
N(z) \leq z^{\frac{1}{k}+\theta}
$$

Proof. If $t \leq z^{1 / k}$, let $m_{1}(t)=t$. Now suppose that $t>z^{1 / k}$ and decompose $t=q_{1} q_{2} \cdots q_{r}$ into its prime powers $q_{1}>\cdots>q_{r}$. By assumption, $q_{1}<t^{\theta}$. Consider $q_{1} \cdots q_{j} \leq z^{1 / k}$ with $j$ maximal. Then $m_{1}(t):=q_{1} \cdots q_{j+1}$ lies in the interval $\left(z^{1 / k}, z^{1 / k+\theta}\right]$. In this way we may split $t$ into $l_{t} \leq k$ pairwise coprime factors

$$
t=q_{1} q_{2} \cdots q_{r}=m_{1}(t) \cdots m_{l_{t}}(t)
$$

with each $m_{i}(t) \leq z^{1 / k+\theta}$.

Now observe each $t \in T$ has some factor $m_{i}(t)$ which is distinct from all other factors $m_{j}(s), s \in T \backslash\{t\}$. Indeed, if not, then each factor of $t$ has $m_{i}(t)=m_{j_{i}}\left(t_{i}\right)$ for some $t_{i} \in T \backslash\{t\}$ (not neccessarily distinct). And since the factors $m_{i}(t)$ are pairwise coprime,

$$
t=m_{1}(t) \cdots m_{l_{t}}(t) \mid \operatorname{lcm}\left[t_{1}, \ldots, t_{l_{t}}\right]
$$

contradicting $T$ as lcm $k$-primitive.

Hence we have a 1-1 map $g: T \rightarrow \mathbb{N}$ via $g(t)=m_{i}(t)$. And since $m_{i}(t) \leq z^{\frac{1}{k}+\theta}$, we conclude $|T|=|g(T)| \leq z^{\frac{1}{k}+\theta}$.

We now fix a choice for $\theta=\theta_{k}$. Let

$$
\theta_{k}=\frac{1}{p_{k}} \text { for } k \neq 3 \text { and } \theta_{3}=\frac{1}{8}
$$

Further, let $\nu_{k}=1 / \theta_{k}$, so that

$$
\nu_{k}=p_{k} \text { for } k \neq 3 \text { and } \nu_{3}=8 .
$$

With these choices we have

$$
\lambda_{k}=2.4 \prod_{j \leq k}\left(1-\theta_{j}\right)
$$

Note that if $Q(t)<t^{\theta_{k}}$, then $t$ must have at least $\nu_{k}+1$ distinct prime factors. Let $P(t)$ denote the largest prime dividing $t$, so that

$$
p_{\nu_{k}+1} \leq P(t) \leq Q(t)<t^{\theta_{k}} \text { which implies } t>p_{\nu_{k}+1}^{\nu_{k}} .
$$

Thus, with $\theta=\theta_{k}, \nu=\nu_{k}$, and $\lambda>\frac{1}{k}+\theta$,

$$
\sum_{\substack{t \in T \\ Q(t)<t^{\theta}}} \frac{1}{t^{\lambda}}=\int_{p_{\nu+1}^{\nu}}^{\infty} \frac{\lambda}{z^{1+\lambda}} N(z) d z \leq \frac{\lambda}{\lambda-\frac{1}{k}-\theta} p_{\nu+1}^{-\nu\left(\lambda-\frac{1}{k}-\theta\right)},
$$

by partial summation and Lemma 5 . 
Lemma 6. Let $k \geq 2$ and let $T$ be an lcm $k$-primitive set of composite numbers. Decompose $T=T^{\prime} \cup T^{\prime \prime}$, where $t \in T^{\prime \prime}$ if there exists another $s \in T$ with $Q(t) \mid$ s; else $t \in T^{\prime}$. Define the map $f: T \rightarrow \mathbb{N}$ via

$$
f(t)= \begin{cases}Q(t) & t \in T^{\prime} \\ t / Q(t) & t \in T^{\prime \prime}\end{cases}
$$

Then $f$ is 1 to 1 and $f(T)$ is an lcm $(k-1)$-primitive set. Further, the members of $f\left(T^{\prime}\right)$ are pairwise coprime proper prime powers.

Proof. First, the map $f$ is $1-1$. Indeed, suppose $f(t)=f\left(t^{\prime}\right)$ for some $t, t^{\prime} \in T$. If $t \in T^{\prime}$ then $Q(t) \nmid t^{\prime}$, in particular $f(t)=Q(t) \neq f\left(t^{\prime}\right) \in\left\{Q\left(t^{\prime}\right), t^{\prime} / Q\left(t^{\prime}\right)\right\}$. Similarly, if $t \in T^{\prime \prime}$ then $Q(t) \mid s$ for some $s \in T \backslash\{t\}$. Thus $1=\operatorname{gcd}(Q(t), t / Q(t))=\operatorname{gcd}\left(Q(t), t^{\prime} / Q\left(t^{\prime}\right)\right)$ implies

$$
t=Q(t) \cdot \frac{t}{Q(t)}=Q(t) \cdot \frac{t^{\prime}}{Q\left(t^{\prime}\right)} \mid \operatorname{lcm}\left[s, t^{\prime}\right]
$$

Thus lcm 2-primitivity of $T$ forces $t=t^{\prime}$. Hence $f$ is indeed 1-1.

Next suppose $f(T)$ is not lcm $(k-1)$-primitive. Then there exist $t \in T$ and $t_{1}, . ., t_{k-1} \in$ $T \backslash\{t\}$ such that

$$
f(t) \mid \operatorname{lcm}\left[f\left(t_{1}\right), \ldots, f\left(t_{k-1}\right)\right] .
$$

If $t \in T^{\prime}$ then $f(t)=Q(t)$ is a prime power, so by above $Q(t) \mid f\left(t_{i}\right)$ for some index $i$. Thus $Q(t) \mid t_{i} \in T \backslash\{t\}$, which contradicts $t \in T^{\prime}$.

Similarly if $t \in T^{\prime \prime}$, then $Q(t) \mid s$ for some $s \in T \backslash\{t\}$, and so $1=\operatorname{gcd}(Q(t), t / Q(t))$ gives

$$
t=Q(t) \cdot \frac{t}{Q(t)}=Q(t) f(t) \mid \operatorname{lcm}\left[s, t_{1}, \ldots, t_{k-1}\right]
$$

contradicting $T$ as lcm $k$-primitive. Hence $f(T)$ is indeed lcm $(k-1)$-primitive. That the members of $f\left(T^{\prime}\right)$ are pairwise coprime follows from $f\left(T^{\prime}\right)$ being a primitive set of prime powers. That the members of $f\left(T^{\prime}\right)$ are proper prime powers follows from the fact that if $Q(t)$ is prime, then by (3.3),$T_{Q(t)}$ has at least 2 elements, and so $t \in T^{\prime \prime}$.

Let $T_{\theta}=\left\{t \in T: Q(t) \geq t^{\theta}\right\}$. We apply Lemma [6 to $T=T_{\theta}$. Thus, by the induction hypothesis on the lcm $(k-1)$-primitive set $f\left(T_{\theta}\right)$, for $\lambda^{\prime}:=\lambda_{k-1}=\frac{\lambda_{k}}{1-\theta}$ we have

$$
\sum_{t \in T_{\theta}} f(t)^{-\lambda^{\prime}}=\sum_{t \in T^{\prime}} Q(t)^{-\lambda^{\prime}}+\sum_{t \in T^{\prime \prime}}(t / Q(t))^{-\lambda^{\prime}}=\sum_{d \in f\left(T_{\theta}\right)} d^{-\lambda^{\prime}} \leq \sum_{p \leq Y} p^{-\lambda^{\prime}} .
$$

Now if $Q(t) \geq t^{\theta}$, then $t / Q(t) \leq t^{(1-\theta)}$ so that $t^{-\lambda} \leq(t / Q(t))^{-\lambda /(1-\theta)}=(t / Q(t))^{-\lambda^{\prime}}$. Thus by the above,

$$
\begin{aligned}
\sum_{t \in T_{\theta}} t^{-\lambda}=\sum_{t \in T^{\prime}} t^{-\lambda}+\sum_{t \in T^{\prime \prime}} t^{-\lambda} & \leq \sum_{t \in T^{\prime}} Q(t)^{-\lambda}+\sum_{t \in T^{\prime \prime}}(t / Q(t))^{-\lambda /(1-\theta)} \\
& \leq \sum_{t \in T^{\prime}}\left(Q(t)^{-\lambda}-Q(t)^{-\lambda^{\prime}}\right)+\sum_{p \leq Y} p^{-\lambda^{\prime}}
\end{aligned}
$$

Thus,

$$
\sum_{t \in T_{\theta}} t^{-\lambda}-\sum_{p \leq Y} p^{-\lambda}<\sum_{p \leq Y}\left(\left(p^{-2 \lambda}-p^{-2 \lambda^{\prime}}\right)-\left(p^{-\lambda}-p^{-\lambda^{\prime}}\right)\right)=: S(Y)
$$


using that $f\left(T^{\prime}\right)$ is a set of pairwise coprime proper prime powers and $\mathcal{P}(T) \subset[1, Y]$. Note that from Lemma 4 we may assume that $Y \geq p_{k}$.

Claim 1: The sequence $S\left(p_{j}\right)$ for $j \geq k$ is decreasing, so if $S\left(p_{k}\right)<0$, then $S(Y)<0$ for all $Y \geq p_{k}$.

Indeed, the terms in $S(Y)$ are of the form $h(y, z)=y-z-\left(y^{2}-z^{2}\right)$, where $y=p^{-\lambda^{\prime}}$ and $z=p^{-\lambda}$. Note that $h(y, z)=(y-z)(1-(y+z))$ and we have $0<y<z$. Further, $p^{-\lambda} \leq \frac{1}{3}$ for $p \geq p_{k}$ and $k \geq 3$, which follows from Lemma 2 and a short calculation. Thus, for $p \geq p_{k}$, the terms in $S(Y)$ are negative, establishing Claim 1.

Claim 2: For $k \geq 3$ we have $S\left(p_{k}\right)<0$ and for $k \geq 200$ we have $S\left(p_{k}\right)<-0.015 / \log p_{k}$.

We verify this directly for $3 \leq k \leq 199$, so assume now that $k \geq 200$. Let $F(\lambda)=$ $\sum_{p \leq p_{k}}\left(p^{-2 \lambda}-p^{-\lambda}\right)$ so that $S\left(p_{k}\right)=F(\lambda)-F\left(\lambda^{\prime}\right)$. By the mean value theorem, there exists some $\xi \in\left(\lambda, \lambda^{\prime}\right)$ with

$$
\begin{aligned}
F(\lambda)-F\left(\lambda^{\prime}\right) & =\left(\lambda-\lambda^{\prime}\right) F^{\prime}(\xi)=\left(\lambda-\lambda^{\prime}\right) \sum_{p \leq p_{k}}\left(p^{-\xi} \log p-2 p^{-2 \xi} \log p\right) \\
& =-\theta \lambda^{\prime} \sum_{p \leq p_{k}}\left(p^{-\xi}-2 p^{-2 \xi}\right) \log p<-\theta \lambda^{\prime} \sum_{p \leq p_{k}}\left(p^{-\lambda^{\prime}}-2 p^{-2 \lambda}\right) \log p .
\end{aligned}
$$

Recall that $\theta=\theta_{k}, \lambda=\lambda_{k}$, and $\lambda^{\prime}=\lambda_{k-1}$. Using Lemma 3, we thus have

$$
\begin{aligned}
S\left(p_{k}\right)=F(\lambda)-F\left(\lambda^{\prime}\right) & <-\theta \lambda^{\prime}\left(p_{k}^{1-\lambda^{\prime}}\left(1-\frac{1}{\log p_{k}}\right)-\frac{2.03248}{1-2 \lambda} p_{k}^{1-2 \lambda}\right) \\
& =-\lambda^{\prime} p_{k}^{-\lambda}\left(p_{k}^{\lambda-\lambda^{\prime}}\left(1-\frac{1}{\log p_{k}}\right)-\frac{2.03248}{1-2 \lambda} p_{k}^{-\lambda}\right) .
\end{aligned}
$$

We use $1-2 \lambda>0.587, p_{k}^{\lambda-\lambda^{\prime}}>1-1 / p_{k}$, and $e^{-1.5}<p_{k}^{-\lambda}<e^{-1.45}$, which follows from Lemma 2, to get

$$
S\left(p_{k}\right)<-\frac{0.015}{\log p_{k}}, \quad \text { for } \quad k \geq 200,
$$

completing the proof of Claim 2 .

By (3.6) and (3.7),

$$
I_{\lambda}=\sum_{\substack{t \in T \\ Q(t)<t^{\theta}}} t^{-\lambda}+\sum_{\substack{t \in T \\ Q(t) \geq t^{\theta}}} t^{-\lambda}-\sum_{p \leq Y} p^{-\lambda}<\frac{\lambda}{\lambda-\frac{1}{k}-\theta} p_{\nu+1}^{-\nu\left(\lambda-\frac{1}{k}-\theta\right)}+S(Y) .
$$

Note though that if $Y<p_{\nu+1}$, then the first term does not appear, so Claims 1 and 2 prove that $I_{\lambda}<0$. So, assume that $Y=p_{\nu+1}$ in (3.6). We check numerically that $I_{\lambda}<0$ for $3 \leq k \leq 199$.

It remains to show that $I_{\lambda}<0$ for $k \geq 200$. Note that if $k \geq 200$, then

$$
\lambda-\frac{1}{k}-\theta>\frac{1.4}{\log p_{k}}, \quad \frac{\lambda}{\lambda-\frac{1}{k}-\theta}<1.05,
$$

using Lemma 3. Thus,

$$
\frac{\lambda}{\lambda-\frac{1}{k}-\theta} p_{\nu+1}^{-\nu\left(\lambda-\frac{1}{k}-\theta\right)}<1.05 p_{p_{k}+1}^{-1.4 p_{k} / \log p_{k}}<1.05 p_{k}^{-1.4 p_{k} / \log p_{k}}=1.05 e^{-1.4 p_{k}}
$$


As a function of $p_{k}$ this expression is much smaller than $0.015 / \log p_{k}$, in fact, this is so for $p_{k} \geq 5$. Thus, (3.8) shows that $I_{\lambda}<0$ for $k \geq 200$. This completes the proof.

\section{TheOrEM FOR lcm $k$-PRIMITIVE SETS}

In this section we prove Theorem 3 . The proof largely follows from the proof for $k$-primitive sets in the previous section. In fact, the only difference is that we start the induction at $k=2$ rather than $k=3$. By Lemma 2 it suffices to prove the following theorem.

Theorem 6. Recall the numbers $\mu_{k}$ in Lemma包. Let $A$ be an lcm k-primitive set. For each $k \geq 1$ we have

$$
\sum_{\substack{a \in A \\ a \leq x}} a^{-\mu_{k}} \leq \sum_{\substack{p \in \mathcal{P}(A) \\ p \leq x}} p^{-\mu_{k}}
$$

for any $x>1$.

First note that since $\tau_{1}<8 / 7=\mu_{1}$, the theorem holds at $k=1$, so we may assume that $k \geq 2$ and the theorem holds for lcm $(k-1)$-primitive sets.

Next note that the various reductions we made in Section 3 hold here, as well as Lemmas 5 and 6. Here we have

$$
\theta_{k}=1 / p_{k} \text { for } k \neq 2, \quad \theta_{2}=1 / 8
$$

so that for all $k \geq 1$,

$$
\mu_{k}=\frac{16}{7} \prod_{j \leq k}\left(1-\theta_{k}\right)
$$

Let $\nu_{k}=1 / \theta_{k}$, so that $\nu_{k}=p_{k}$ for $k \neq 2$ and $\nu_{2}=8$. With these new values, we continue to have (3.6) recorded anew as follows:

$$
\sum_{\substack{t \in T \\ Q(t)<t^{\theta}}} \frac{1}{t^{\mu}}=\int_{p_{\nu+1}^{\nu}}^{\infty} \frac{\mu}{z^{1+\mu}} N(z) d z \leq \frac{\mu}{\mu-\frac{1}{k}-\theta} p_{\nu+1}^{-\nu\left(\mu-\frac{1}{k}-\theta\right)},
$$

where $\mu=\mu_{k}, \theta=\theta_{k}, \nu=\nu_{k}$.

We have the analogue of (3.7), where $\lambda$ is replaced with $\mu=\mu_{k}$ and $\lambda^{\prime}$ is replaced with $\mu^{\prime}=\mu_{k-1}$. In addition, we continue to have Claim 1, checking that $p^{-\mu} \leq \frac{1}{3}$ for $p \geq p_{k}$.

However, Claim 2 needs to be verified. As before, we check that $S\left(p_{k}\right)<0$ for $2 \leq k \leq 199$. Following the argument for $k \geq 200$, we have $1-2 \mu>0.528, p_{k}^{\mu-\mu^{\prime}}>1-1 / p_{k}$, and $e^{-1.7}<p_{k}^{-\lambda}<e^{-1.65}$, again following from Lemma 3. Thus,

$$
\begin{aligned}
S\left(p_{k}\right) & <-\frac{1.65 e^{-1.7}}{\log p_{k}}\left(\left(1-\frac{1}{p_{k}}\right)\left(1-\frac{1}{\log p_{k}}\right)-\frac{2.03248}{0.528} e^{-1.65}\right) \\
& <-\frac{0.035}{\log p_{k}}, \quad \text { for } k \geq 200 .
\end{aligned}
$$

This is somewhat stronger than Claim 2.

We have the analogue of (3.9):

$$
I_{\mu}<\frac{\mu}{\mu-\frac{1}{k}-\theta} p_{\nu+1}^{-\nu\left(\mu-\frac{1}{k}-\theta\right)}+S(Y),
$$


where the first term does not occur if $Y<p_{\nu+1}$. Our goal is to show that $I_{\mu}<0$. Thus, by Claims 1 and 2, we may assume that $Y=p_{\nu+1}$. We then check numerically that the bound in (4.3) is negative for $2 \leq k \leq 199$.

To show that $I_{\mu}<0$ for $k \geq 200$, note that

$$
\mu-\frac{1}{k}-\theta>\frac{1.6}{\log p_{k}}, \quad \frac{\mu}{\mu-\frac{1}{k}-\theta}<1.05
$$

in analogy to what we had before. Thus,

$$
\frac{\mu}{\mu-\frac{1}{k}-\theta} p_{\nu+1}^{-\nu\left(\mu-\frac{1}{k}-\theta\right)}<1.05 e^{-1.6 p_{k}},
$$

which is smaller than before. Hence $I_{\mu}<0$ for $k \geq 200$, which completes the proof.

\section{TheOREM FOR STRONGLY $k$-PRIMITIVE SETS}

In this section we prove Theorem 4 .

As in Section 3 we may assume that $A=T$ consists of composite numbers, for each $p \in \mathcal{P}(T)$ we have $\left|T_{p}\right| \geq 2$, and $\mathcal{P}(T)$ consists of all of the primes up to some point $Y$. Note that since $\tau_{k}^{(\mathrm{s})} \leq \tau_{k}$ for all $k$, Theorem 4 follows from Theorem 5 when $k \leq 38$. Thus, in the sequel, we assume that $k \geq 39$ and that the theorem holds for $k-1$.

For $k \geq 39$, let

$$
\lambda=\lambda_{k}=\frac{3 \log k}{k}, \quad \theta=\theta_{k}=1-\frac{\lambda_{k}}{\lambda_{k-1}} .
$$

A simple calculation shows that

$$
\nu=\nu_{k}:=\frac{1}{\theta_{k}}>\frac{k \log (k-1)}{\log (k-1)-1}>k .
$$

Recall that $P(t)$ denotes the largest prime factor of $t$. Let

$$
T_{0}=\left\{t \in T: P(t)<t^{\theta}\right\} .
$$

We now prove a version of Lemma 5 dealing with $T_{0}$.

Lemma 7. Let $N_{0}(z)$ denote the number of $t \in T_{0}$ with $t \leq z$. Then

$$
N_{0}(z) \leq z^{\frac{1}{k}+\theta}
$$

Proof. Let $t \in T_{0}, t \leq z$. If $t \leq z^{1 / k}$, let $m_{1}(t)=t$. Otherwise, say the prime factorization of $t$ is $p_{1} p_{2} \cdots p_{r}$, where $p_{1} \geq p_{2} \geq \cdots \geq p_{r}$. Let $j$ be minimal with $p_{1} \cdots p_{j}>z^{1 / k}$. Since all of these primes are $<t^{\theta} \leq z^{\theta}$, we have $m_{1}(t):=p_{1} \cdots p_{j} \leq z^{\frac{1}{k}+\theta}$. Continuing in this fashion we obtain a factorization

$$
t=p_{1} p_{2} \cdots p_{r}=m_{1}(t) m_{2}(t) \cdots m_{l_{t}}(t), \quad l_{t} \leq k, \quad \text { each } m_{i}(t) \leq z^{\frac{1}{k}+\theta} .
$$

We claim that each $t$ has at least one factor $m_{i}(t)$ that does not appear in the analogous factorization for any other $t^{\prime} \in T_{0}$. Indeed, if each $m_{i}(t)=m_{j_{i}}\left(t_{i}^{\prime}\right)$ for some $t_{i}^{\prime} \in T_{0} \backslash\{t\}$ with $j_{i} \leq l_{t_{i}^{\prime}}$, then $t \mid t_{1}^{\prime} t_{2}^{\prime} \cdots t_{l_{t}}^{\prime}$, contradicting $T_{0}$ as strongly $k$-primitive. By mapping $t$ to such a unique factor $m_{i}(t)$ we obtain a 1 to 1 function from $T_{0}$ to the integers in $\left(1, z^{\frac{1}{k}+\theta}\right]$, so proving the lemma. 
Because of the change in the definition of $N(z)$ we do not have (3.6). Instead, we argue as follows. Note that every member of $T_{0}$ has at least $\lceil\nu\rceil$ prime factors, counted with multiplicity. Thus, the least element of $T_{0}$ is at least $2^{\nu}$. In addition, the second smallest member of $T_{0}$ must be $\geq 3^{\nu}$. Indeed, if there are two members smaller than this, then $P(t)<t^{\theta}$ implies they are both powers of 2 , and hence $T_{0}$ is not primitive. More generally, using Lemma 4, $T_{0}$ has at most $j$ members smaller than $p_{j+1}^{\nu_{k}}$ for each $j \leq k$. Thus,

$$
\begin{aligned}
\sum_{t \in T_{0}} \frac{1}{t^{\lambda}} & <\sum_{j \leq k} \frac{1}{p_{j}^{\nu \lambda}}+\int_{p_{k+1}^{\nu}}^{\infty} \frac{\lambda}{z^{1+\lambda}} N_{0}(z) d z \\
& <\sum_{j \leq k} \frac{1}{p_{j}^{\nu \lambda}}+\frac{\lambda}{\lambda-\frac{1}{k}-\theta} p_{k+1}^{-\nu\left(\lambda-\frac{1}{k}-\theta\right)}
\end{aligned}
$$

by partial summation and Lemma 7 .

In the next lemma we give a variant of Lemma 6 in a more general setting.

Lemma 8. Let $k \geq 2$ and let $T$ be an arbitrary strongly $k$-primitive set of composite numbers such that for each prime $p \in \mathcal{P}(T),\left|T_{p}\right| \geq 2$. Then the map $f: T \rightarrow \mathbb{N}$ given by $f(t)=t / P(t)$ is 1 to 1 and $f(T)$ is $(k-1)$-primitive.

Proof. Suppose $t, t^{\prime} \in T, t \neq t^{\prime}$, and $f(t)=f\left(t^{\prime}\right)$. Since $\left|T_{P(t)}\right| \geq 2$, there is some $s \in T \backslash\{t\}$ with $P(t) \mid s$. Then

$$
t=P(t) \cdot \frac{t}{P(t)}=P(t) \cdot \frac{t^{\prime}}{P\left(t^{\prime}\right)} \mid s t^{\prime},
$$

contradicting $T$ as strongly 2-primitive. Thus, $f$ is 1 to 1 .

Next, suppose that $f(T)$ is not strongly $(k-1)$-primitive, so that there are $t, t_{1}, \ldots t_{k-1}$ in $T$ with $t \notin\left\{t_{1}, \ldots, t_{k}\right\}$ and

$$
f(t) \mid f\left(t_{1}\right) \cdots f\left(t_{k-1}\right) .
$$

With $P(t) \mid s \neq t$ as above, we have $t \mid s \cdot t_{1} \cdots t_{k-1}$, contradicting $T$ as strongly $k$-primitive. Thus, $f(T)$ is strongly $(k-1)$-primitive, and the proof is complete.

Let $T_{\theta}=T \backslash T_{0}=\left\{t \in T: P(t) \geq t^{\theta}\right\}$. We apply Lemma 8 to $T$, and so restricting the injection $f$ to $T_{\theta}$, we have $f\left(T_{\theta}\right)$ as a $(k-1)$-primitive set. Further, every $t \in T_{\theta}$ has $f(t) \leq t^{1-\theta}$. Thus, $t^{-\lambda} \leq(t / P(t))^{-\lambda /(1-\theta)}$ and by the induction hypothesis on the $(k-1)$ primitive set $f\left(T_{\theta}\right)$,

$$
\sum_{t \in T_{\theta}} t^{-\lambda} \leq \sum_{t \in T_{\theta}}(t / P(t))^{-\lambda^{\prime}}=\sum_{d \in f\left(T_{\theta}^{\prime}\right)} d^{-\lambda^{\prime}} \leq \sum_{p \leq Y} p^{-\lambda^{\prime}}
$$

for $\lambda^{\prime}:=\lambda_{k-1}=\frac{\lambda_{k}}{1-\theta}$.

By way of (5.1), this allows us to replace (3.9) with

$$
I_{\lambda}=\sum_{t \in T} t^{-\lambda}-\sum_{p \leq Y} p^{-\lambda}<\sum_{p \leq p_{k}} p^{-\nu \lambda}+\frac{\lambda}{\lambda-\frac{1}{k}-\theta} p_{k+1}^{-\nu\left(\lambda-\frac{1}{k}-\theta\right)}+\sum_{p \leq Y}\left(p^{-\lambda^{\prime}}-p^{-\lambda}\right),
$$

with the goal as before to show that $I_{\lambda}<0$.

By the mean value theorem, there is some $\xi \in\left(\lambda, \lambda^{\prime}\right)$ with

$$
\sum_{p \leq Y}\left(p^{-\lambda^{\prime}}-p^{-\lambda}\right)=-\left(\lambda^{\prime}-\lambda\right) \sum_{p \leq Y} \frac{\log p}{p^{\xi}}<-\lambda^{\prime} \theta \sum_{p \leq Y} \frac{\log p}{p^{\lambda^{\prime}}} .
$$


Since by Lemma 4 we may assume that $Y \geq p_{k+1}$, it suffices, by Lemma 3 , for us to show that

$$
\sum_{p \leq p_{k}} p^{-\nu \lambda}+\frac{\lambda}{\lambda-\frac{1}{k}-\theta} p_{k+1}^{-\nu\left(\lambda-\frac{1}{k}-\theta\right)}<\lambda^{\prime} \theta p_{k+1}^{1-\lambda^{\prime}}\left(1-\frac{1}{\log p_{k+1}}\right) .
$$

Now $\nu \lambda>3 \log k$, so that

$$
\sum_{p \leq p_{k}} p^{-\nu \lambda}<2^{-3 \log k}+(k-1) 3^{-3 \log k}<k^{-2}+k \cdot k^{-3}=2 k^{-2} .
$$

Using $k \geq 39$ we see that $\nu\left(\lambda-\frac{1}{k}-\theta\right)>3 \log k-2$ and $\lambda /\left(\lambda-\frac{1}{k}-\theta\right)<1.23$, so that

$$
\frac{\lambda}{\lambda-\frac{1}{k}-\theta} p_{k+1}^{-\nu\left(\lambda-\frac{1}{k}-\theta\right)}<1.23 p_{k+1}^{-(3 \log k-2)}<k^{-2} .
$$

So the left side of (5.2) is $<3 k^{-2}$. We now get a lower bound for the right side. Using $k \geq 39$, we have $\lambda^{\prime} \theta>2(\log k) / k^{2}$ and $p_{k+1}^{\lambda^{\prime}}<4.4$. Thus,

$$
\lambda^{\prime} \theta p_{k+1}^{1-\lambda}\left(1-\frac{1}{\log p_{k+1}}\right)>0.79 \frac{2 \log k}{k^{2}} p_{k+1} / 4.4>\frac{0.36 p_{k+1} \log k}{k^{2}}>\frac{0.36 \log ^{2} k}{k},
$$

using that $p_{k+1}>p_{k}>k \log k$. We do indeed have $3 / k^{2}<0.36\left(\log ^{2} k\right) / k$ when $k \geq 39$, so we have (5.2), and the theorem.

\section{ACKNOWLEDGMENTS}

The authors would like to acknowledge the University of Memphis for hosting the 2019 Erdős Lecture Series, during which the initial ideas for this paper and its prequel were conceived. The second named author is supported by a Clarendon Scholarship at the University of Oxford.

\section{REFERENCES}

1. W. D. Banks and G. Martin, Optimal primitive sets with restricted primes, Integers 13 (2013), \#A69, $10 \mathrm{pp}$.

2. T. H. Chan, On sets of integers, none of which divides the product of $k$ others, European J. Comb. 32 (2011), 443-447.

3. T. H. Chan, E. Győri, and A. Sárközy, On a problem of Erdős on integers, none of which divides the product of $k$ others, European J. Comb. 31 (2010), 260-269.

4. T. H. Chan, J. D. Lichtman, and C. Pomerance, A generalization of primitive sets and a conjecture of Erdős, Discrete Analysis 16 (2020), 13 pp.

5. P. Erdős, Note on sequences of integers no one of which is divisible by any other, J. London Math. Soc. 10 (1935), 126-128.

6. P. Erdős, On sequences of integers no one of which divides the product of two others and on some related problems, Tomsk. Gos. Univ. Ucen. Zap. 2 (1938), 74-82.

7. P. Erdős, On the integers having exactly $k$ prime factors, Ann. of Math. 49 (1948), 53-66.

8. P. Erdős, A. Sárközy, and E. Szemerédi, On an extremal problem concerning primitive sequences, J. London Math. Soc. 42 (1967), 484-488.

9. J. D. Lichtman, Almost primes and the Banks-Martin conjecture, Journal of Number Theory, 211 (2020), $513-529$.

10. J. D. Lichtman and C. Pomerance, The Erdős conjecture for primitive sets, Proc. Amer. Math. Soc., Series B, 6 (2019), 1-14. 
11. P. P. Pach and Cs. Sándor, Multiplicative bases and an Erdős problem, Combinatorica, 38 (2018), no. 5, 1175-1203.

12. J. B. Rosser and L. Schoenfeld, Approximate formulas for some functions of prime numbers, Illinois J. Math. 6 (1962), 64-94.

Department of Mathematics, Kennesaw State University, Kennesaw, Ga 30144

Email address: thchan6174@gmail.com

Mathematical Institute, University of Oxford, Oxford, OX2 6GG, UK

Email address: jared.d.lichtman@gmail.com

Department of Mathematics, Dartmouth College, Hanover, NH 03755

Email address: carl.pomerance@dartmouth.edu 